\title{
Silicon Photonic MEMS Add-Drop Filter
}

Hamed Sattari(1), Alain Yuji Takabayashi(1), Pierre Edinger(2), Peter Verheyen(3), Kristinn B. Gylfason(2), Wim Bogaerts ${ }^{(3,4)}$, Niels Quack ${ }^{(1)}$

(1) École Polytechnique Fédérale de Lausanne, 1015 Lausanne, Switzerland, hamed.sattari@epfl.ch

(2) KTH Royal Institute of Technology, School of Electrical Engineering and Computer Science, Micro and Nanosystems, Stockholm, Sweden

(3) Interuniversity Microelectronics Centre (IMEC), Leuven, Belgium

(4) Ghent University-imec, Photonics Research Group, Department of Information Technology, Ghent,

Belgium

Abstract We demonstrate a compact add-drop filter based on a MEMS ring resonator implemented in IMEC's iSIPP50G silicon photonics platform. The device exhibits a port extinction of $20 \mathrm{~dB}$ and a port isolation of $>50 \mathrm{~dB}$, upon actuation range of $0 \mathrm{~V}$ to $27 \mathrm{~V}$.

\section{Introduction}

Silicon photonics has over the past decades emerged as a mature technology for high performance Photonic Integrated Circuits (PIC). Standardized silicon photonics technology platforms benefit from the well-established fabrication processes, building on the experience of CMOS electronics microfabrication, and have fuelled an abundance of high-performance devices available to PIC designers as standard library components. Among them, add-drop filters based on optical ring resonators have proven successful for manipulating spectral channels in Wavelength Division Multiplexing (WDM) circuits.

Active add-drop filters in standard silicon photonics platforms typically exploit thermo-optic or plasma dispersion effects. Thermally controlled add-drop filters provide a wide range of tunability $(>10 \mathrm{~nm})$ but with a slow ms response time $e^{[1]}$. Their high power consumption and thermal crosstalk limit the number of components that can be integrated in a single circuit. Add-drop filters based on the plasma dispersion effect provide ns response time with no substantial crosstalk ${ }^{[2]}$. Nevertheless, such filters typically have a limited tuning range and exhibit excessive optical losses due to photoncarrier scattering in the active region of the component.

Recently, microelectromechanical systems (MEMS) technology has been recognized as an excellent path to augment standard silicon photonics. Benefits include the possibility for lowpower operation, large effective index tunability, and compatibility with standard silicon photonics platform fabrication processes ${ }^{[3]}$. Hitherto, silicon photonic MEMS add-drop filters have been realized by implementing movable waveguides and ring/disk resonators ${ }^{[4]-[6]}$. Nevertheless, such previous demonstrations required custom photonics technology.
We here present a tunable MEMS add-drop filter, integrated in IMEC's iSiPP50G standard platform, requiring only a few additional postprocessing steps to release the MEMS devices. The release process is wafer-level compatible and does not impact the performance of standard library components as we demonstrate by including several such devices next to the MEMS add-drop filter. In addition to the excellent performance of the MEMS add-drop filter reported in this paper, the integration of MEMS in a standard photonics platform presents a milestone in the scaling of silicon photonic MEMS to large scale circuits $[7],[8]$.

\section{MEMS Filter Working Principle and Design}

A schematic representation of the silicon photonic MEMS add-drop filter is shown in Fig.1.

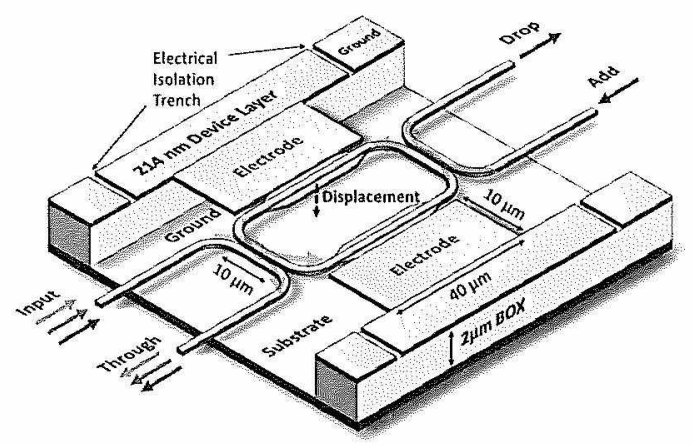

Fig. 1: Schematic representation of the MEMS add-drop filter (not to scale). The device is tunable by actuating the vertically movable suspended ring resonator.

The filter consists of two suspended directional couplers connected in a loop to form a suspended ring resonator, and a pair of parallel plate electrostatic actuators provide vertical movement. By applying a voltage between the device layer and silicon substrate (i.e., the parallel plate actuators), the ring is pulled down. Consequently, the air gaps between the suspended waveguides increase, which 
modulates the coupling efficiency and the fraction of power transmitted to the drop and through ports. The suspended MEMS ring resonator is composed of three sections: fully etched waveguides for the coupler region, shallow etched waveguides for the anchoring region, and tapered waveguides to ensure adiabatic transition between the waveguide types. The two suspended directional couplers are $10 \mu \mathrm{m}$ long, and the bent waveguides have a bending radius of $5 \mu \mathrm{m}$. Waveguide widths are $450 \mathrm{~nm}$ and 650 $\mathrm{nm}$ for the fully-etched and shallow-etched waveguide regions, respectively, and the airgap between the coupling waveguides is $150 \mathrm{~nm}$ in the unactuated state. As defined by the iSiPP50G standard process, the final waveguide height is $214 \mathrm{~nm}$ and the shallow waveguide section has a ridge height of $70 \mathrm{~nm}$. At $45 \mu \mathrm{m} \times 75 \mu \mathrm{m}$, this device has a small footprint, advantageous for large-scale integration and has four ports: input, through, add, and drop. The optical carrier enters the circuit from the input port, couples to the resonator in the first coupler and circulates in the MEMS ring resonator before coupling through the second directional coupler and transferring to the drop port. To add a wavelength to the spectrum, an additional optical carrier is launched into the add port and is transferred to the through port after traveling in the resonator. The MEMS ring resonator supports filtering of carrier wavelengths in the telecommunication C-band with a free spectral range of $5 \mathrm{~nm}$.

\section{Fabrication Process}

The fabrication process builds upon the standard iSiPP50G process at IMEC [8]. Areas where the MEMS devices are placed have the back-end-ofline (BEOL) stack opened up as part of a standard processing step, which provides access to the silicon device layer. Subsequently, a set of post-processing steps performed at the EPFL Center of Micronanotechnology (CMi) releases these MEMS devices by selectively removing the buried oxide layer underneath them. The main steps of this process for the photonic MEMS release are shown in Fig. 2.

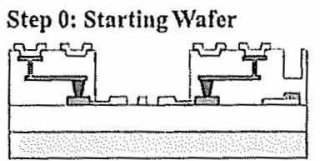

Step 1: Alumina ALD
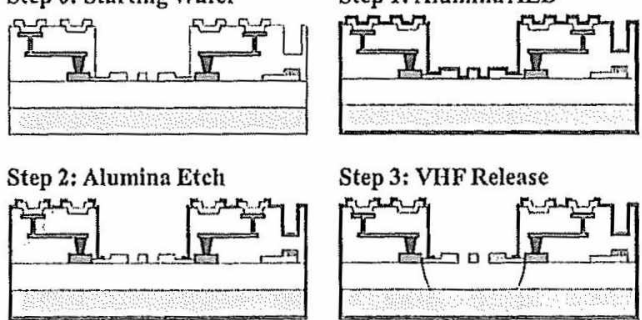

Step 3; VHF Release

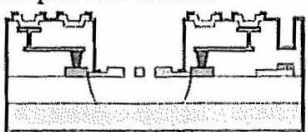

$\square \mathrm{Si} \square \mathrm{SiO}_{2} \square$ Doped Si $\square \mathrm{WW} \mathrm{Cu} \square \mathrm{AlCu}$

Fig. 2: Post-processing steps for releasing the silicon photonic MEMS components.
Note that the wafer contains the full stack present in the standard silicon photonics platform and includes various standard components such as heaters, electro-optical modulators and photodetectors integrated alongside the novel photonic MEMS components.

As the first step in the post-processing sequence, we deposit $20 \mathrm{~nm}$ of alumina using an atomic layer deposition (ALD) process (BENQ TSF200) to provide a conformal passivation of the sample. Next, we define access windows above the MEMS by maskless lithography and etch the alumina selectively by dry etching. In the final MEMS release step, the $2 \mu \mathrm{m}$ buried oxide underneath the device layer is selectively removed by vapour phase hydrofluoric acid (VHF), which leads to suspended, movable structures.
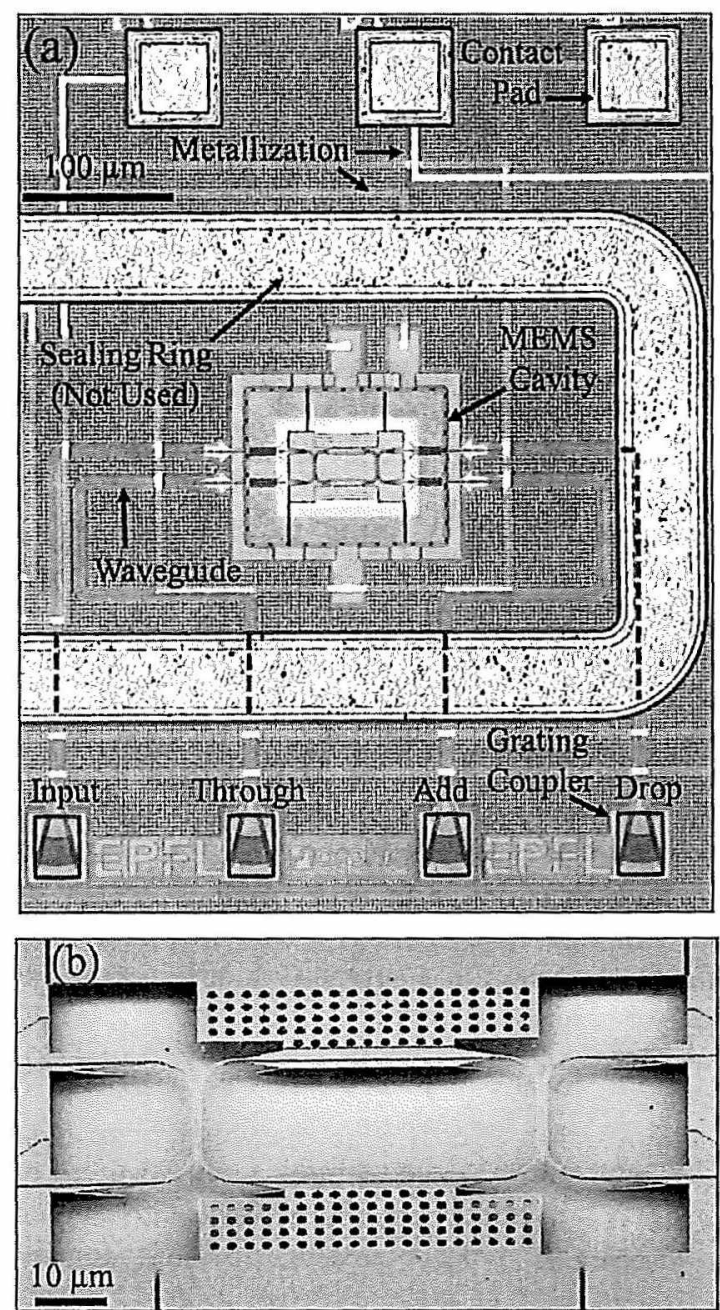

Fig. 3: (a) Optical microscope image of the chip area after vapour phase hydrofluoric acid MEMS release. (b) SEM image of the add-drop filter (tilted view), showing well defined suspended actuators and waveguides.

Fig. 3.a shows an optical microscope image of the chip just after the MEMS release process. In Fig.3.b, the Scanning Electron Microscope 
(SEM) image of the MEMS add-drop filter demonstrates the compatibility of the MEMS release process with the standard silicon photonics platform, yielding well-defined suspended actuators and well-aligned and parallel directional coupler waveguides.

\section{Characterization and Discussion}

The characterization setup is shown schematically in Fig.4. An array of single mode fibers connects the tunable laser, chip, and the power sensor. The optical signal couples into/of the chip by means of library-standard, vertical grating couplers. A set of electrical probes on micromanipulators enable the active tuning of the MEMS add-drop filter by applying electrical signals to the actuator.

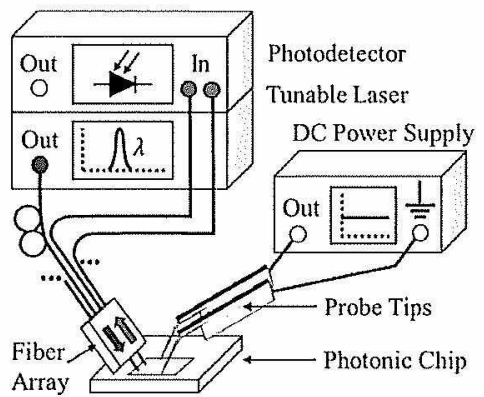

Fig. 4: Schematic of the characterization setup.

The drop and the through ports' transmission spectra for the passive ( $0 \mathrm{~V}$ bias) and selected active (27 V bias) state are presented in Fig. 5.a. The MEMS ring resonator exhibits $\sim 5 \mathrm{~nm}$ of Free Spectral Range (FSR). In the passive state, we observe a port extinction of $\sim 20 \mathrm{~dB}$ at a resonance wavelength of $\lambda=1552 \mathrm{~nm}$, with a loaded quality factor of $\sim 25^{\prime} 000$. In the active state, with a bias of $27 \mathrm{~V}$, we observe transfer of the optical signal to the through port, while maintaining a port extinction of $>20 \mathrm{~dB}$. We note a resonance shift of $0.5 \mathrm{~nm}$ upon the actuation voltage of $27 \mathrm{~V}$ which can be attributed to change in the optical path in the ring resonator upon actuation.

The actuation curves for selected onresonance $(\lambda=1552 \mathrm{~nm})$ and off-resonance $(\lambda=$ $1549 \mathrm{~nm}$ ) wavelengths are plotted in Fig.5.b., confirming high port isolation of $\sim 50 \mathrm{~dB}$ and $57 \mathrm{~dB}$ for the on- and off-resonance wavelengths, respectively.

The insertion loss of the MEMS tunable adddrop filter is predicted by FDTD simulations not to exceed $0.4 \mathrm{~dB}$. However, from the characterization results (Fig.5.a) the drop port at the passive state at the resonance exhibits an extra loss of $\sim 7 \mathrm{~dB}$. This additional loss can be attributed to the loss in the two couplers and the MEMS ring resonator. A precise determination of the insertion loss that requires dedicated monitoring structures, such as sequence of couplers, has not been included in the current study.
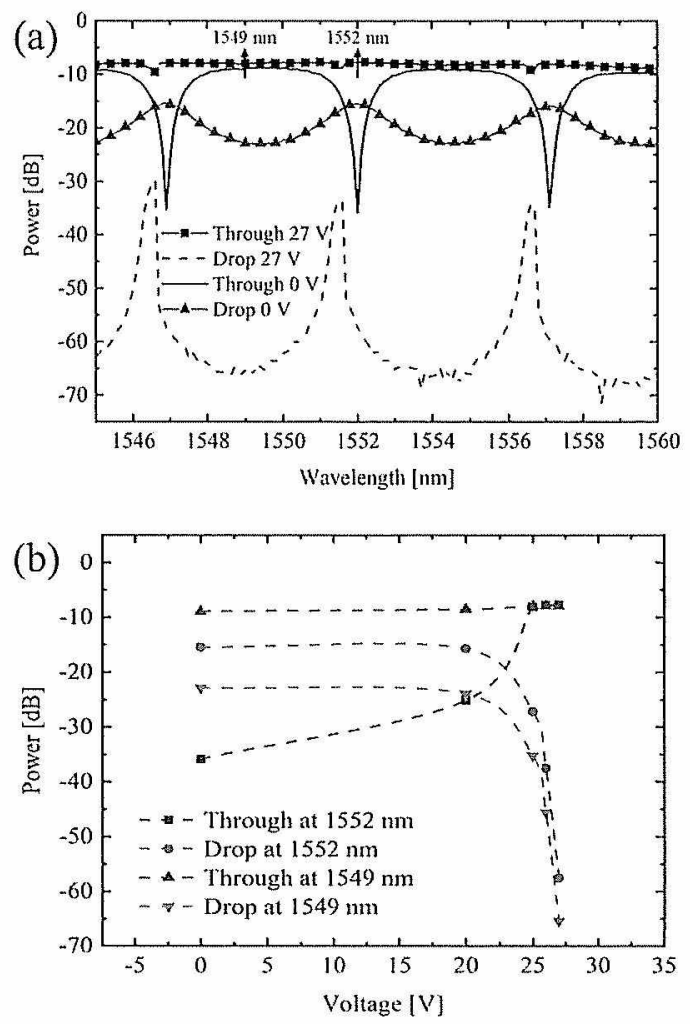

Fig. 5: (a) Power spectra of the drop and through ports of the MEMS add-drop filter for passive $(0 \mathrm{~V})$ and actuated (27 V) states. (b) Actuation curve of the device at $\lambda=1552 \mathrm{~nm}$ (on-resonance) and $\lambda=1549 \mathrm{~nm}$ (offresonance).

\section{Conclusions}

We report for the first time the design, fabrication, and characterization of a compact MEMS adddrop filter implemented within the IMEC iSiPP50G standard silicon photonics platform extended with MEMS release post processing. The device consists of a vertically movable ring resonator with an FSR of $5 \mathrm{~nm}$. Port extinctions of $20 \mathrm{~dB}$ and $>50 \mathrm{~dB}$ are reported at the passive and active states of the device, respectively. With a low actuation voltage (below $27 \mathrm{~V}$ ), a compact footprint of $45 \mu \mathrm{m} \times 75 \mu \mathrm{m}$, and robust mechanical properties, this add-drop filter can serve as an advantageous building block for integrated WDM applications.

\section{Acknowledgements}

This project has received funding from the European Union's Horizon 2020 research and innovation program under grant No. 780283 (MORPHIC www.h2020morphic.eu). H. Sattari acknowledges funding from the Hasler Foundation under grant No. 17008, and Niels Quack from the Swiss National Science Foundation under grant No. 183717. 


\section{Bnffi. Pierparto}

We2H - 6: Mode-group divislon multiplexing for provisioning in SDM networks

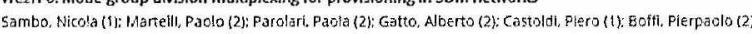

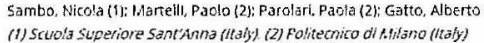

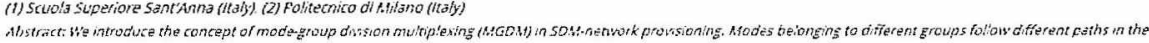

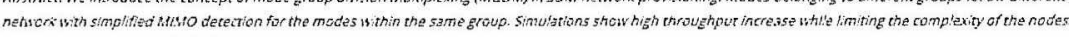

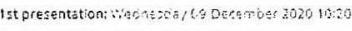

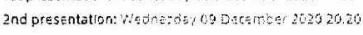

Bogaerts, wirm

TU28-2: Silicon Photonic MEMS Add-Drop Filter

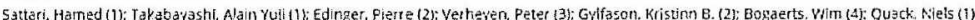

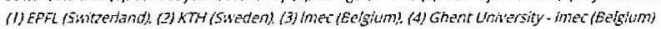

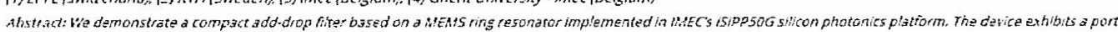

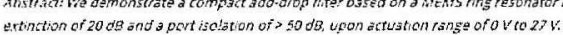

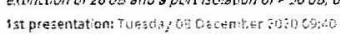

Ind presentation: TUa:

Bogaerts, Yлm

Tust-1: (Tutorial) Programmable integrated Photontes

Bogaers, Wim; Chen, Xiangieng; Zand, Iman; Wang. Mí, Deng, Hong; Von iseghem, Lukas: Rahlim, Addul: Khan Umar

Ghent University - imer (Belyium)

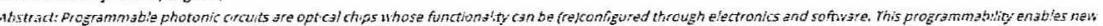

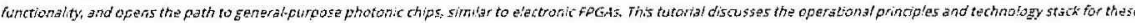

chps:

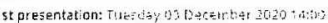

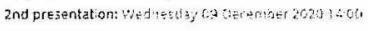

\section{Bogrs. Adonts}

We10-4: Performance and Complexity Evaluation of Recurrent Neural Hetwork Badels for Fibre Nonlinear Equalization in Digital Coherent Systerns Deligiennidis. Stavros (1): Mesaritakis, Charis (2): Rogris. Adonis (1)

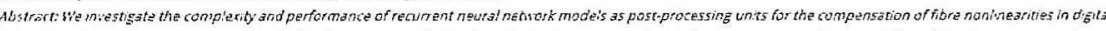

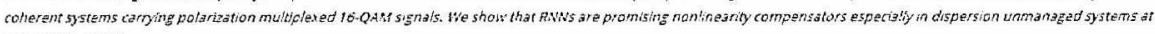

ressanable comp!e

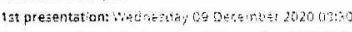

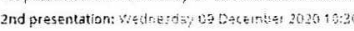

